\title{
Appetitive and Aversive Olfactory Learning in Humans Studied Using Event-Related Functional Magnetic Resonance Imaging
}

\author{
Jay A. Gottfried, John O'Doherty, and Raymond J. Dolan \\ Wellcome Department of Imaging Neuroscience, Institute of Neurology, London, WC1N 3BG, United Kingdom
}

\begin{abstract}
We combined event-related functional magnetic resonance imaging (fMRl) with olfactory classical conditioning to differentiate the neural responses evoked during appetitive and aversive olfactory learning. Three neutral faces [the conditioned stimuli $(\mathrm{CS}+)$ ] were repetitively paired with pleasant, neutral, or unpleasant odors [the unconditioned stimuli (UCS)] in a partial reinforcement schedule. A fourth face was never paired to odor [the nonconditioned stimulus (CS-)]. Learning-related neural activity, comparing unpaired (face only) CS + stimuli with CS-, showed valence-independent activations in rostral and caudal orbitofrontal cortex (OFC). Medial OFC responded to the appetitive (app) CS + , whereas lateral OFC responded to the aversive (av) CS+. Within nucleus accumbens, neural responses showed divergent activation profiles that increased
\end{abstract}

with time in response to the appCS+ but decreased in response to the avCS + . In posterior amygdala, responses were elicited by the appCS+, which habituated over time. In temporal piriform cortex, neural responses were evoked by the avCS + , which progressively increased with time. These results highlight regional and temporal dissociations during olfactory learning and imply that emotionally salient odors can engender cross-modal associative learning. Moreover, the findings suggest that the role of human primary (piriform) and secondary olfactory cortices transcends their function as mere intermediaries of chemosensory information processing.

Key words: olfaction; odor; emotion; conditioning; associative learning; neuroimaging; $\mathrm{fMRl}$; orbitofrontal cortex; nucleus accumbens; amygdala; piriform cortex
Behavior is powerfully influenced by the biological salience of environmental stimuli. Odors can impact on behavioral states by virtue of their associations with threat, food, and sex. For example, bombykol, a volatile pheromone secreted by female silkworm moths, will drive potential mates miles upwind in hot pursuit (Carde and Mafra-Neto, 1997). Despite a conspicuous phylogenetic decline in olfactory prowess, humans can exhibit behavioral vulnerability to odors in certain instances (MacFarlane, 1975; Beauchamp and Maller, 1977; Baron, 1988; Stern and McClintock, 1998).

How the brain encodes and recalls behaviorally meaningful information can be investigated through associative learning paradigms. Classical (Pavlovian) conditioning is one such example in which a previously neutral item [the conditioned stimulus (CS +$)$ ] becomes predictive of an emotionally salient item [the unconditioned stimulus (UCS)] through repeated pairings. In aversive conditioning the UCS is often represented by an electric shock or loud sound, whereas foods, liquids, or drugs commonly substitute for an appetitive (reward-based) UCS. Animal studies indicate that the amygdala is critical to aversive (fear) conditioning (LeDoux, 2000), whereas dopaminergic mesolimbic circuitry has been implicated in appetitive learning (Schultz, 1998; Kalivas and Nakamura, 1999). Human neuroimaging studies of conditioning have essentially focused on aversive paradigms (Buchel et al.,

\footnotetext{
Received July 16, 2002; revised Sept. 23, 2002; accepted Sept. 27, 2002.

This research was supported by a Physician Postdoctoral Fellowship from the Howard Hughes Medical Institute (J.A.G.) and by a Wellcome Trust Programme Grant (R.J.D.). We thank P. Aston, E. Featherstone, O. Josephs, and J. S. Winston, as well as the radiology staff of the Wellcome Department of Imaging Neuroscience.

Correspondence should be addressed to Dr. Jay A. Gottfried, Wellcome Department of Imaging Neuroscience, Functional Imaging Laboratory, 12 Queen Square, London WC1N 3BG, UK. E-mail: j.gottfried@fil.ion.ucl.ac.uk.

Copyright (C) 2002 Society for Neuroscience $0270-6474 / 02 / 2210829-09 \$ 15.00 / 0$
}

1998; LaBar et al., 1998; Morris et al., 2001), with few studies addressing reward learning (O'Doherty et al., 2002).

Given the hedonic primacy of odor perception (Schiffman, 1974), odors can act as rewarding or aversive UCS stimuli in conditioning experiments. In aversion therapy for overeaters, desserts become endowed with the aversive properties of an unpleasant odor after multiple pairings (Frohwirth and Foreyt, 1978), although this effect is short-lived (Cole and Bond, 1983). When an odor UCS is paired with a visual (face) CS+, both subjective preferences of the faces and physiological indices of conditioning are significantly altered (Todrank et al., 1995; Hermann et al., 2000). In the olfactory neuroimaging literature, few studies have explored higher-order aspects of odor processing [although see Savic et al. (2000), Royet et al. (2001), and Dade et al. (2002)], and none has attempted to characterize the brain regions involved in olfactory associative learning.

In this report we describe the use of event-related functional magnetic resonance imaging (fMRI) techniques to delineate the neural substrates underlying human olfactory conditioning. By manipulating the affective properties of the odor UCS, we indexed alternate modes of conditioning within the same experiment: appetitive learning in the case of a pleasant odor and aversive learning in the case of an unpleasant odor. We predicted that variations in the emotional valence of learning would elicit differential responses in orbital frontal cortex (OFC), amygdala, and ventral striatum, regions strongly implicated in appetitive and aversive learning. We also hypothesized that olfactory structures involved in low-level sensory processing [as defined in a companion paper in this issue (Gottfried et al., 2002)] would show modulation of their response as a function of associative learning.

\section{MATERIALS AND METHODS}

The detailed methodology has been reported in the accompanying paper (Gottfried et al., 2002), and only distinct aspects are described here. 
Subjects. Informed consent was obtained from 17 healthy right-handed subjects ( 10 women; mean age, 23 years), although data from 2 subjects (1 woman) was discarded because of technical problems. The study was approved by the joint National Hospital for Neurology and Neurosurgery and Institute of Neurology Ethics Committee.

Stimuli and experiment. Subjects participated in an olfactory form of classical conditioning that was similar to previous experiments in our laboratory (Buchel et al., 1998; Morris et al., 2001). Here, a series of neutral faces was paired with odors that varied in pleasantness. Four neutral faces (two male, two female) comprised the one nonconditioned $(\mathrm{CS}-)$ and the three conditioned (CS+) stimuli and were projected to the subjects as they lay inside the scanner. Three odors comprised the pleasant ( $8 \%$ vanillin), neutrally valenced ( $0.1 \%$ phenethyl alcohol), and unpleasant (5\% 4-methyl-pentanoic acid) UCS stimuli. These were delivered using a four-channel computer-controlled olfactometer suitable for the fMRI environment (Lorig et al., 1999). Under $50 \%$ reinforcement, only one-half of all faces (CS+) were paired with their corresponding odor, resulting in seven discrete conditions: (1) "appetitive" face, paired with pleasant odor $\left(\operatorname{appCS}+_{\mathrm{P}}\right) ;(2)$ appetitive face, unpaired $\left(\operatorname{appCS}+_{U}\right) ;(3)$ "neutral” face, paired with neutral odor $\left(\right.$ ntCS $\left.+_{\mathrm{p}}\right)$; (4) neutral face, unpaired $\left(\mathrm{ntCS}+_{\mathrm{U}}\right)$; $(5)$ "aversive" face, paired with unpleasant odor $\left(\operatorname{avCS}+_{\mathrm{P}}\right) ;(6)$ aversive face, unpaired $\left(\operatorname{avCS}+_{\mathrm{U}}\right)$; and $(7)$ face never paired with odor (CS-). This scheme allowed us to model responses to the appetitive and aversive $\mathrm{CS}+$ without interference from the odor UCS.

During the task, subjects made a forced-choice push-button response regarding facial gender. Trial onset was marked by the appearance of a face. After $500 \mathrm{msec}$, the brightening of a dull-red cross-hair cued subjects to sniff, and the olfactometer was simultaneously triggered to deliver odor or control air, depending on the condition type. In this manner, subjects completed a sniff on each trial, regardless of odor presence. The stimulus duration of faces, sniff cues, and odor pulses was $750 \mathrm{msec}$ in all cases. Thus, the CS+ and UCS overlapped for $250 \mathrm{msec}$. The intertrial interval was $7.5 \mathrm{sec}$, and stimulus presentation was randomized. Each event type was repeated 25 times, except for the CS- $(50$ times), for an experimental duration of $25 \mathrm{~min}$. Subject-specific respiratory patterns (sniffs) were monitored on-line via corrugated breathing belts (Siemens, Erlangen, Germany) coupled to a differential pressure sensor (Honeywell, Morristown, NJ).

Conditioning index. Subject reaction times (RTs), which have been used successfully in our laboratory as a reliable index of conditioning (Critchley et al., 2002), were measured to gauge the efficacy of learning. RTs were recorded using Cogent 2000 software (Wellcome Department of Imaging Neuroscience, London, UK). Off-line analysis was performed using Matlab 6.0 (The Mathworks Inc., Natick, MA). Condition-specific RTs for each subject were averaged over three successive temporal blocks of the experiment. Differences between unpaired CS + and CSconditions were calculated for each valence level (aversive, appetitive, neutral) and pooled across subjects. RT data from six subjects (appetitive, aversive events) and eight subjects (neutral events) were unavailable because of technical complications.

Image acquisition. Gradient echo $\mathrm{T} 2 *$-weighted echoplanar images (EPI) were acquired with blood-oxygen level-dependent (BOLD) contrast on a 2-Tesla Siemens Vision MRI scanner (Siemens, Erlangen, Germany), using an optimized sequence to reduce signal dropout in orbitofrontal and ventral temporal lobes (Deichmann and Turner, 2002). There were 33 slices per volume (1.8 mm thickness, $1.2 \mathrm{~mm}$ gap), which covered $\sim 80 \%$ of the whole brain, and they were acquired continuously every $2.31 \mathrm{sec}$. Images were spatially realigned (Friston et al., 1995a), slice-time corrected, normalized, and smoothed with an $8 \mathrm{~mm}$ kernel. T1-weighted anatomical images were also obtained from each subject and co-registered to the mean normalized EPI.

Analysis of learning-evoked responses. Event-related fMRI data were analyzed in SPM99 (Wellcome Department of Imaging Neuroscience) (Friston et al., 1995b) using a random-effects model as described in the companion paper (Gottfried et al., 2002), with the following exceptions. Regressors of interest included the six CS + conditions, plus the CScondition divided randomly into two time series of 25 events each (designated "appCS-" and "avCS-"). The estimation of two independent CS - baselines was necessary to implement conjunction analysis (see below), although it is important to note that appCS- and avCSrepresented experimentally identical event types. To model learning, the design incorporated condition $\times$ time interactions, using a time constant of one-quarter session length $(372 \mathrm{sec})$ similar to that used previously in neuroimaging studies of conditioning from our laboratory (Buchel et al.,
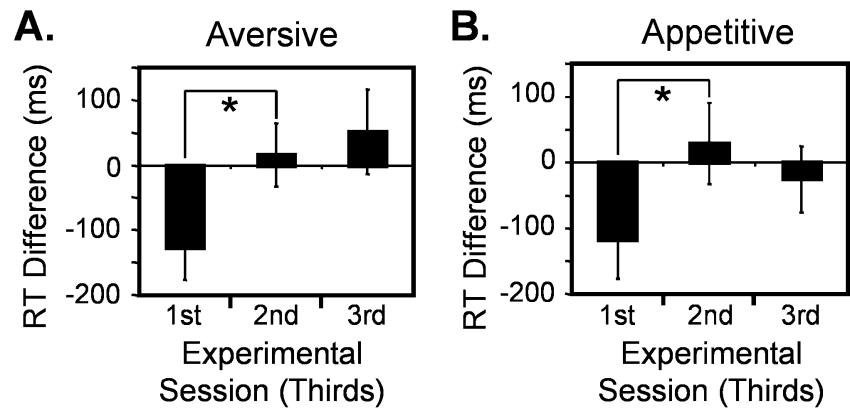

Figure 1. Differential reaction times. Condition-specific RTs were averaged across subjects over each of three successive blocks of the experiment. $A$, RT differences between an aversive $\mathrm{CS}+_{\mathrm{U}}$ and $\mathrm{CS}-($ mean \pm SEM). Significant between-block differences were set at $p<0.05$ and are indicated by asterisk. $B$, RT differences between an appetitive $\mathrm{CS}+{ }_{\mathrm{U}}$ and CS - . Significance, $p<0.05$.

1998; Morris et al., 2001). Neural responses specific to learning were isolated by contrasting the unpaired CS + events with their designated CS- baselines.

Activations common to both appetitive and aversive learning were tested using conjunction analysis (Price and Friston, 1997). Subjectspecific parameter estimates were calculated for four effects of interest: appCS $+_{\mathrm{U}}$, avCS $+_{\mathrm{U}}$, appCS - , and avCS - . (This process was repeated for the four time-modulated regressors.) These estimates from all 15 subjects were then entered into a repeated-measures ANOVA, using nonsphericity correction. By testing appropriate pairs of linear contrasts, we were able to identify learning-related responses independent of specific valence. As detailed in the accompanying paper (Gottfried et al. 2002), a descriptive model of the data was also constructed to depict time courses of activation within significant (accumbens) voxels derived from the primary model.

We report activations surviving a threshold of $p<0.001$ uncorrected in regions predicted a priori, including brain areas implicated in associative learning (OFC, amygdala, nucleus accumbens, ventral tegmental area) (O'Doherty et al., 2002a). In medial temporal sites involved in olfactory sensory processing (piriform cortex) and learning (hippocampus), the threshold was set at $p<0.005$ uncorrected. Correction for multiple comparisons was performed across small volumes of interest (Worsley et al., 1996) by constructing binary anatomical masks of amygdala, nucleus accumbens, OFC (including medial, lateral, anterior, and posterior subdivisions), and piriform cortex to limit the effective search space. The statistical threshold used to report these activations was set at $p<0.05$ corrected for small volumes of interest or, where indicated, for whole-brain volume. All voxels of interest are reported in Montreal Neurological Institute (MNI) coordinate space.

\section{RESULTS}

\section{Behavioral}

Subjects responded more slowly to the $\operatorname{avCS}{ }_{U}$ face compared with the CS- during early phases of aversive learning, but this differential effect habituated later in the experiment (Fig. 1A). The main effect across experimental blocks approached significance $(n=9$ subjects; $F=3.52$; df $=2 ; p=0.054$; one-way repeated-measures ANOVA). Post hoc paired $t$ tests revealed that RTs were significantly slower in the first compared with the second block $(p<0.05)$ and approached significance in a comparison of first and third blocks $(p=0.068)$. Similar differential responses were seen during appetitive learning in response to the appCS ${ }_{U}$ face (Fig. $1 B$ ). There was a significant main effect across the three sessions $(n=9$ subjects; $F=3.69$; df $=2 ; p<$ 0.05; repeated-measures ANOVA), and post hoc comparisons revealed the RTs were significantly more delayed in the first than the second block $(p<0.05)$. By comparison, there was no significant main difference of RTs across experimental blocks for 
A. i.

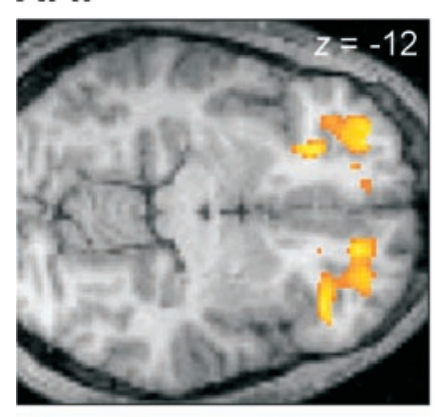

B. i.

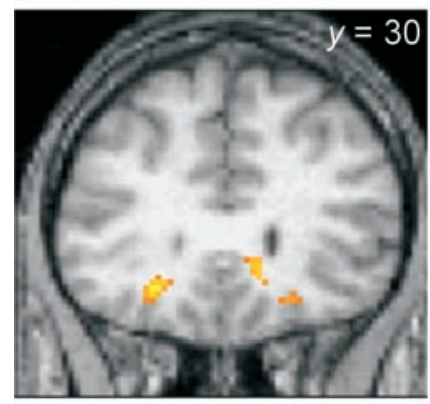

ii.

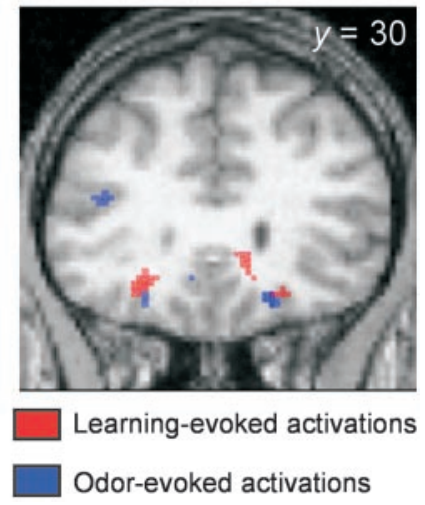

Figure 2. Valence-independent learning. $A$, Rostral orbitofrontal cortex. The SPM (threshold, $p<0.001$ ) is overlaid on the axial $(i)$ and coronal (ii) sections of a subject's normalized T1-weighted scan and depicts extensive bilateral activations in rostral areas of orbitofrontal cortex. In this and all subsequent figures, the right side of the brain corresponds to the right side of the image. $B$, Caudal orbitofrontal cortex. $i$, Neural activations in caudal orbitofrontal cortex are superimposed on a normalized T1-weighted coronal image (threshold, $p<0.001$ ). $i i$, The learningevoked activations depicted in $i$ are illustrated in red (threshold, $p<$ $0.001)$ and overlap the odor-evoked activations derived from the companion study (Gottfried et al., 2002), which are shown in blue (threshold, $p<$ $0.005)$.

neutral learning ( $n=7$ subjects; $F=3.21$; df $=1.17 ; p=0.114$; repeated-measures ANOVA, Greenhouse-Geisser adjusted).

\section{Neuroimaging}

Valence-independent olfactory learning

Sustained responses. To highlight brain regions involved in olfactory learning, regardless of specific valence, we tested for regions showing a common response during both forms of learning based on the conjunction of $\left(\operatorname{appCS}+_{U}-\operatorname{appCS}-\right)$ and $\left(\operatorname{avCS}+_{U}-\right.$ avCS-). This analysis revealed confluent activations in OFC with distinct peaks that encompassed medial, anterior, posterior, and lateral orbital gyri (Fig. $2 A$, Table 1 ). The most significant peaks were in the right rostral OFC [medial: $x=14, y=46, z=-18$; $Z=5.85$; and anterior: $28,46,-6 ; Z=4.79$; both $p<0.05$ corrected for whole-brain volume), although activations were also detected on the left (medial: $-18,46,-12 ; Z=3.45 ; p<0.001$ uncorrected; and anterior: $-28,50,-12 ; Z=4.36 ; p<0.05$ small-volume corrected (SVC) over the region of interest]. Learning-evoked responses in bilateral centroposterior OFC $(-24,30,-12 ; Z=4.41 ; p<0.05 \mathrm{SVC}$; and $22,32,-16 ; Z=3.65$; $p=0.052 \mathrm{SVC}$ ) closely overlapped caudal OFC regions defined previously in our companion study (Gottfried et al., 2002) as "secondary olfactory" cortex (at 20, 30, -20, and -24, 34, -16)
(Fig. 2B). Significant effects were also observed in lateral orbital gyri (right: $34,36,-10 ; Z=4.38$; and $38,34,-12 ; Z=4.28$; left: $-30,54,-10 ; Z=4.41$; and $-34,46,-12 ; Z=3.87 ; p<0.05$ SVC). Outside of OFC, the only other neural responses elicited by the learning conjunction were in retrosplenial cortex, which closely bordered significance $(0,-48,10 ; Z=4.75 ; p=0.051$ whole-brain corrected).

Time-dependent responses. We also performed conjunctions between condition $\times$ time interactions [namely, $\left(\operatorname{appCS}+_{U} \times\right.$ time - appCS $-\times$ time $)$ and $\left(\operatorname{avCS}+_{U} \times\right.$ time - avCS $-\times$ time)] to detect time-dependent changes in neural activity common to appetitive and aversive learning. Common learningrelated activations that showed progressive increases over time (response potentiation) were evident in ventromedial prefrontal cortex (PFC) $(4,34,-14 ; Z=3.20 ; p<0.001$ uncorrected), anterolateral $\mathrm{OFC}(32,46,-12 ; Z=3.17 ; p<0.001$ uncorrected $)$, and cerebellum $(-12,-40,-46 ; Z=4.81 ; p<0.05$ whole-brain corrected) (Table 1). No significant time-dependent decreases were found in this analysis.

\section{Appetitive olfactory learning}

Sustained responses. We next tested for the main effect of appetitive learning in the contrast $\left(\operatorname{appCS}+_{U}-\right.$ appCS-), which showed several activation foci in medial OFC $(18,44,-16 ; Z=$ 4.03; $p<0.05$ SVC; $16,50,-10 ; Z=3.80 ; p<0.001$ uncorrected; and 10, 36, $-14 ; Z=3.56 ; p<0.001$ uncorrected) (Fig. $3 A$ ). These responses extended into gyrus rectus and medial PFC. An additional focus of activation was observed in anterior OFC (30, 42, $-10 ; Z=3.60 ; p<0.001$ uncorrected; $p=0.058$ SVC). The direct contrast $\left(\operatorname{appCS}+_{U}-\operatorname{avCS}+_{U}\right)$ demonstrated bilateral activity in ventral striatum (Fig. $3 B$ ). Neural activations in the right hemisphere bordered the ventral caudate head $(14,6,2 ; Z=$ 3.12; $p<0.001$ uncorrected), whereas left-sided responses were localized to nucleus accumbens $(-8,4,-4 ; Z=3.07 ; p=0.051$ SVC). Bilateral responses were observed in dorsomedial areas of posterior amygdala, bounded superiorly and posteriorly by ventral pallidum (left: $-20,-14,-12 ; Z=3.37 ; p<0.001$ uncorrected; and right: $16,-10,-12 ; Z=2.94 ; p<0.005$ uncorrected), and also in a more ventral region of the right medial temporal lobe $(14,-12,-22 ; \mathrm{Z}=3.07 ; p<0.005$ uncorrected) that extended anteriorly into amygdala (Fig. $3 C$, Table 2).

Time-dependent responses. Time-dependent modulations of neural activity specific to appetitive learning were examined using condition $\times$ time interactions [that is, $\left(\operatorname{appCS}+_{U} \times\right.$ time) relative to (appCS $-\times$ time)]. Learning-related increases (response potentiation) were evident in nucleus accumbens $(14,16,-12$; $Z=3.82 ; p<0.05$ SVC) (Fig. 4) and ventromedial PFC (4, 34, $-16 ; Z=3.24 ; p<0.001$ uncorrected). Activations in right cerebellar hemisphere were also elicited $(26,-46,-34 ; Z=5.06$; $p<0.05$ whole-brain corrected). A direct comparison between $\left(\right.$ appCS $+_{U} \times$ time $)$ and $\left(\right.$ avCS $+_{U} \times$ time $)$ revealed significant habituating responses in bilateral anterior hippocampus (right: $-36,-14,-20 ; Z=3.12 ; p<0.001$ uncorrected; and left: -32 , $-14,-16 ; Z=3.07 ; p<0.005$ uncorrected) and in a region of right medial temporal lobe $(-16,-12,-24 ; Z=3.05 ; p<0.005$ uncorrected) that was contiguous with posterior ventromedial amygdala (at $y=-10)$ (Table 2).

\section{Aversive olfactory learning}

Sustained responses. By testing the contrast $\left(\operatorname{avCS}{ }_{U}-\operatorname{avCS}-\right)$, we determined neural activity evoked by aversive olfactory learning. This revealed significant activations in rostral OFC with 
Table 1. Regions commonly activated by olfactory learning, independent of valence

\begin{tabular}{|c|c|c|c|c|c|}
\hline \multirow[b]{2}{*}{ Brain region } & \multicolumn{3}{|c|}{ MNI coordinates $(\mathrm{mm})$} & \multirow[b]{2}{*}{ Peak $Z$} & \multirow[b]{2}{*}{$p$ value } \\
\hline & $x$ & $y$ & $z$ & & \\
\hline \multicolumn{6}{|l|}{ Main effect (conjunction) } \\
\hline Right medial rostral OFC & 14 & 46 & -18 & 5.85 & $<0.05^{* *}$ \\
\hline Left medial rostral OFC & -18 & 46 & -12 & 3.45 & $<0.001$ \\
\hline Right anterior OFC & 28 & 46 & -6 & 4.79 & $<0.05^{* *}$ \\
\hline Left anterior OFC & -28 & 50 & -12 & 4.36 & $<0.05^{*}$ \\
\hline Right centroposterior OFC & 22 & 32 & -16 & 3.65 & $0.052^{*}$ \\
\hline Left centroposterior OFC & -24 & 30 & -12 & 4.41 & $<0.05^{*}$ \\
\hline \multirow[t]{2}{*}{ Right lateral OFC } & 34 & 36 & -10 & 4.38 & $<0.05^{*}$ \\
\hline & 38 & 34 & -12 & 4.28 & $<0.05^{*}$ \\
\hline \multirow[t]{2}{*}{ Left lateral OFC } & -30 & 54 & -10 & 4.41 & $<0.05^{*}$ \\
\hline & -34 & 46 & -12 & 3.87 & $<0.05^{*}$ \\
\hline Retrosplenial cortex & 0 & -48 & 10 & 4.75 & $0.051^{*}$ \\
\hline \multicolumn{6}{|c|}{ Condition $\times$ time interaction (conjunction): time-dependent increases } \\
\hline Right ventromedial PFC & 4 & 34 & -14 & 3.20 & $<0.001$ \\
\hline Right antero-lateral OFC & 32 & 46 & -12 & 3.17 & $<0.001$ \\
\hline Left cerebellar hemisphere & -12 & -40 & -46 & 4.81 & $<0.05^{* *}$ \\
\hline
\end{tabular}

* Corrected for small volume of interest; ** corrected for whole-brain volume.

appetitive olfactory learning. $A$, In the main effect of appetitive learning $\left(\operatorname{appCS}_{\mathrm{U}}-\operatorname{appCS}-\right), \mathrm{BOLD}$ activity was significantly increased within medial and anterior orbitofrontal cortex. The SPMs are superimposed on coronal (i) and axial (ii) sections of a T1weighted scan (threshold, $p<0.001$ ). $B$, $C$, Neural activations in bilateral nucleus accumbens $(B)$ and posterior amygdala/medial temporal lobe $(C)$ were revealed in the direct contrast of $\left(\operatorname{appCS}+_{U}-\operatorname{avCS}+_{U}\right)$ and are depicted in coronal $(i)$ and axial (ii) formats (threshold, $p<0.005$ ).
A. i.

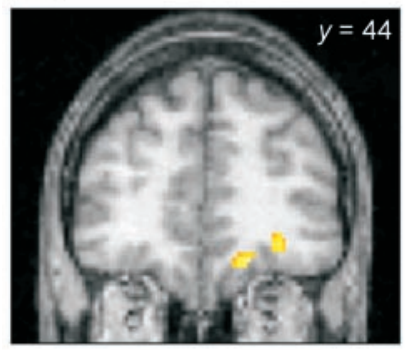

A. ii.

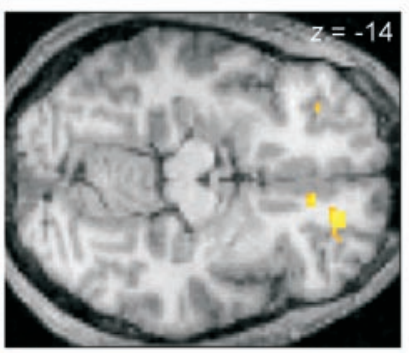

B. i.

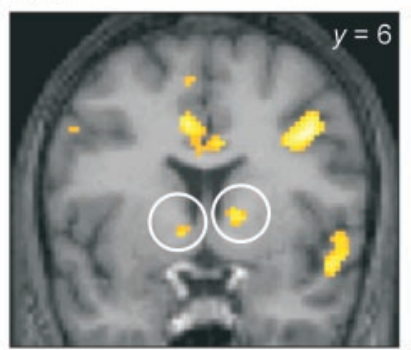

C. i.

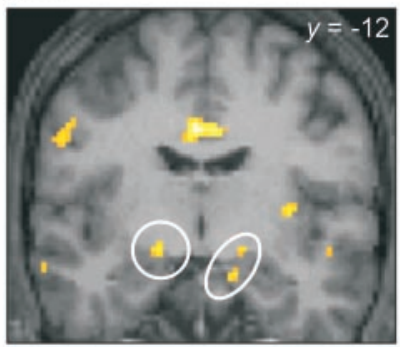

B. ii.

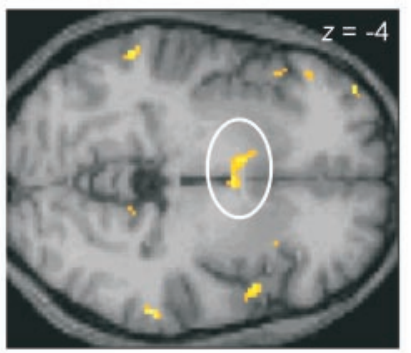

C. ii.

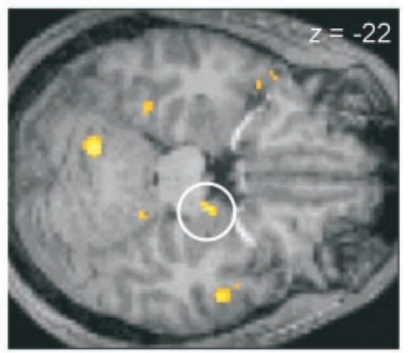

peaks in left lateral $(-32,52,-12 ; Z=4.19 ; p<0.05$ SVC) (Fig. $5 A)$ and right medial $(14,46,-18 ; Z=3.88 ; p<0.001$ uncorrected) orbital gyri. No significant responses were observed in the direct comparison of avCS ${ }_{\mathrm{U}}$ and appCS ${ }_{\mathrm{U}}$ conditions.

Time-dependent responses. Time-dependent neural responses during aversive learning were examined by contrasting condition $\times$ time interactions between avCS ${ }_{U}$ and its baseline (avCS-). Time-dependent increases in activity (response potentiation) were observed in left $\mathrm{OFC}(-18,22,-14 ; Z=3.99 ; p=$ $0.058 \mathrm{SVC})$ and in left temporopolar cortex $(-26,6,-38 ; Z=$ 3.26; $p<0.001$ uncorrected) (Fig. 5B,i, Table 3). This latter response extended caudally into periamygdaloid and posterior (temporal) piriform cortices $(y=2)$. Similar response enhancements in anterior mesiotemporal structures (peak: $-30,6,-38$; $Z=4.74 ; p<0.001$ uncorrected) were identified in the direct contrast of time-modulated aversive $\left(\operatorname{avCS}{ }{ }_{U} \times\right.$ time $)$ and ap- petitive $\left(\right.$ appCS $+_{\mathrm{U}} \times$ time) learning conditions (Fig. 5B,ii.). Conversely, the only brain region that showed a significant decline in activity over time (response habituation) was nucleus accumbens (-10, 16, $-12 ; Z=3.10 ; p<0.05$ SVC) (Fig. 5C). This habituating profile was opposite to the temporal pattern evoked by appetitive learning in nucleus accumbens, where neural activity increased progressively over time (Fig. 4). These divergent responses are depicted in Figure 6.

\section{DISCUSSION}

Our results indicate that emotionally salient odors can drive associative learning processes. We identified regional and temporal dissociations between appetitive and aversive conditioning within several key areas implicated in studies of associative learning, including OFC, nucleus accumbens, and amygdala. In addi- 
Table 2. Regions activated by appetitive olfactory learning

\begin{tabular}{|c|c|c|c|c|c|}
\hline \multirow[b]{2}{*}{ Brain region } & \multicolumn{3}{|c|}{ MNI coordinates $(\mathrm{mm})$} & \multirow[b]{2}{*}{ Peak $Z$} & \multirow[b]{2}{*}{$p$ value } \\
\hline & $x$ & $y$ & $z$ & & \\
\hline \multicolumn{6}{|l|}{$\left(\operatorname{appCS}+_{U}-\operatorname{appCS}-\right)$} \\
\hline \multirow[t]{3}{*}{ Right medial OFC } & 18 & 44 & -16 & 4.03 & $<0.05^{*}$ \\
\hline & 16 & 50 & -10 & 3.80 & $<0.001$ \\
\hline & 10 & 36 & -14 & 3.56 & $<0.001$ \\
\hline Right anterior OFC & 30 & 42 & -10 & 3.60 & $0.058^{*}$ \\
\hline \multicolumn{6}{|l|}{$\left(\operatorname{appCS}+_{U}-\operatorname{avCS}+_{U}\right)$} \\
\hline Right ventral striatum & 14 & 6 & 2 & 3.12 & $<0.001$ \\
\hline Left nucleus accumbens & -8 & 4 & -4 & 3.07 & $<0.051^{*}$ \\
\hline Left pallidum/dorsomedial amygdala & -20 & -14 & -12 & 3.37 & $<0.001$ \\
\hline Right uncus/ventromedial amygdala & 14 & -12 & -22 & 3.07 & $<0.005$ \\
\hline Right dorsomedial amygdala & 16 & -10 & -12 & 2.94 & $<0.005$ \\
\hline \multicolumn{6}{|c|}{$\left(\operatorname{appCS}+_{U}-\operatorname{appCS}-\right) \times$ time: time-dependent increases } \\
\hline Right nucleus accumbens & 14 & 16 & -12 & 3.82 & $<0.05^{*}$ \\
\hline Right ventromedial PFC & 4 & 34 & -16 & 3.24 & $<0.001$ \\
\hline Right cerebellar hemisphere & 26 & -46 & -34 & 5.06 & $<0.05^{* *}$ \\
\hline \multicolumn{6}{|c|}{$\left(\operatorname{appCS}+_{U}-\operatorname{avCS}+_{U}\right) \times$ time: time-dependent decreases } \\
\hline Left anterior hippocampus & -32 & -14 & -16 & 3.34 & $<0.001$ \\
\hline Right anterior hippocampus & 36 & -14 & -20 & 3.12 & $<0.001$ \\
\hline Left uncus/ventromedial amygdala & -16 & -12 & -24 & 3.05 & $<0.005$ \\
\hline
\end{tabular}

* Corrected for small volume of interest; ** corrected for whole-brain volume.

A.

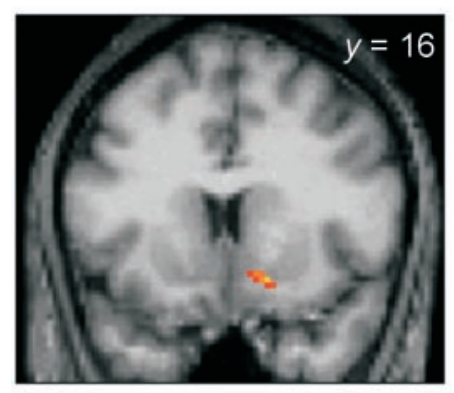

B.

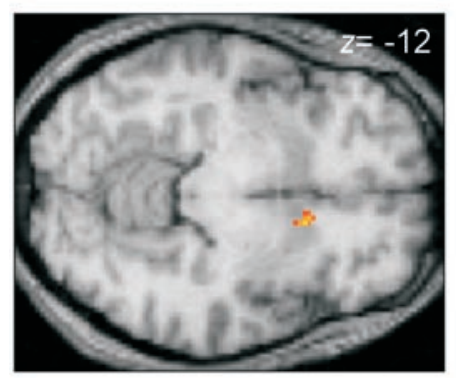

C.

$(14,16,-12)$

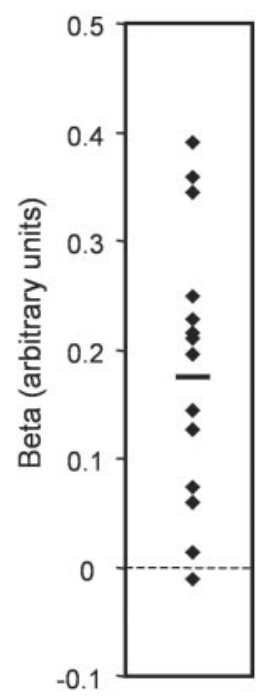

Figure 4. Time-dependent response enhancements in nucleus accumbens elicited by appetitive learning. The main effect of $\left(\operatorname{appCS}+_{U} \times\right.$ time) relative to (appCS $-\times$ time) demonstrates increasing activity in right nucleus accumbens over the course of the experiment. SPMs are displayed on coronal $(A)$ and axial $(B)$ slices of a T1-weighted scan (threshold, $p<0.005)$. $C$, The contrasts of parameter estimates from the peak voxel within right nucleus accumbens are plotted for each subject, and the mean value $(0.17)$ is indicated by a short horizontal bar.

tion, the activation of brain areas previously found to participate in low-level odor processing, such as piriform cortex and caudal OFC, implies that these regions play an active role in the transfer of affective value between olfactory and visual modalities.

The behavioral data (Fig. 1) show that to the extent that conditioning occurs, it is mostly completed by the second block of trials. This temporal pattern resembles those obtained in previous imaging investigations using autonomic markers, whereby learningbased differences were expressed in the early phase of conditioning (Buchel et al., 1998; LaBar et al., 1998). In addition, these behavioral profiles mirror the time course (exponential function, onequarter time constant) used to model learning-specific responses in the fMRI data. The fact that significant differential responses to the neutral $\left(\mathrm{ntCS}^{+} \mathrm{U}\right)$ stimulus were not seen supports the idea that successful olfactory learning is contingent on odor valence rather than the presence of odor per se. We should point out that the absence of a significant behavioral effect in the third block of trials suggests that other processes (e.g., fatigue, boredom) may be interfering with olfactory conditioning. By itself this does not invalidate the responses that are seen, but raises the possibility that other learning-specific responses might have been missed.

Neural responses common to both appetitive and aversive learning were evident in widespread regions of rostral and caudal OFC (Fig. 2). The extent of these activations indicates that OFC is critical to the learning of olfactory-visual associations, regardless of specific valence. These findings concur with animal studies implicating OFC in many higher-order aspects of olfactory learning (Schoenbaum and Eichenbaum, 1995; Critchley and Rolls, 1996; Schoenbaum et al., 1998). Although studies of human patients with orbitofrontal lesions have advanced the idea that OFC is essential to odor discrimination (Potter and Butters, 1980; Zatorre and Jones-Gotman, 1991), the impact of these lesions on odor learning has not been addressed. Likewise, although OFC is consistently detected in olfactory neuroimaging studies (Zald and Pardo, 2000), its role in more complex processes has received limited attention (O’Doherty et al., 2000; Savic et al., 2000; Dade et al., 2001, 2002; Royet et al., 2001). Our study provides evidence that higher-order olfactory computations, specifically those related to learning, are engaged by human OFC. 
Figure 5. Neural activations evoked by aversive olfactory learning. $A$, The main effect of aversive learning $\left(\mathrm{avCS}_{\mathrm{U}}-\right.$ avCS - ) showed responses in left lateral orbitofrontal cortex. The SPM is overlaid on coronal $(i)$ and axial (ii) sections of a T1-weighted image and thresholded at $p<0.001 . B, i$, In the contrast of condition $\times$ time interactions $\left(\operatorname{avCS}+_{U} \times\right.$ time - avCS $-\times$ time), significant response increases were seen in anteromedial temporal lobe extending into temporal piriform cortex and periamygdaloid areas (threshold, $p<0.005$ ). $i i$, Similar time-dependent enhancements of activity were detected in the direct comparison between $\left(\operatorname{avCS}+_{U} \times\right.$ time $)$ and (appCS ${ }_{\mathrm{U}} \times$ time) (threshold, $p<$ $0.001)$. $C, i$, Time-dependent response decreases were observed in nucleus accumbens in the contrast of $\left(\operatorname{avCS}{ }_{U} \times\right.$ time - avCS $-\times$ time) (threshold, $p<$ 0.005). $\ddot{i}$, The contrasts of parameter estimates from the peak accumbens voxel are plotted for each subject, along with the mean value $(0.12)$.
A. i.

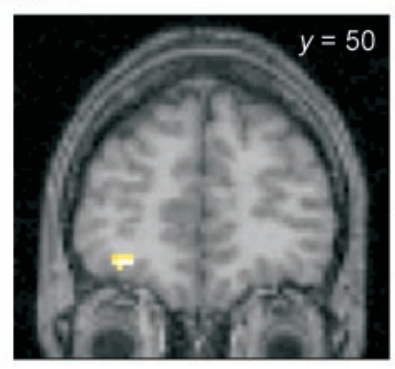

A. ii.

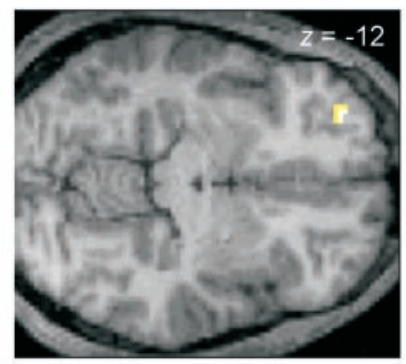

B. i.

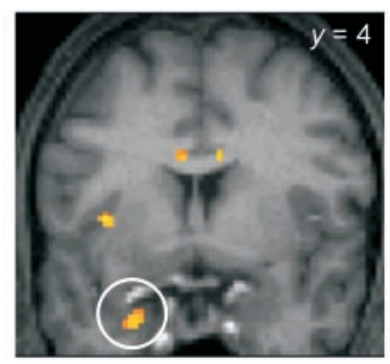

B. ii.

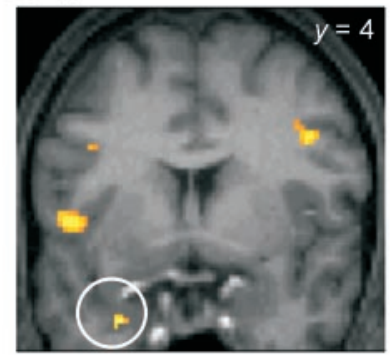

C. i.

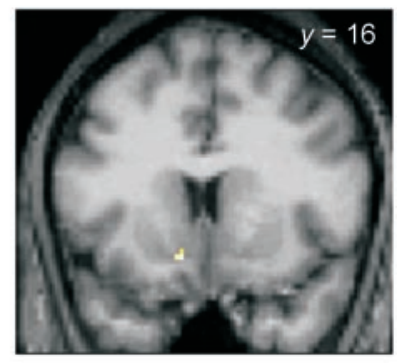

C. ii.

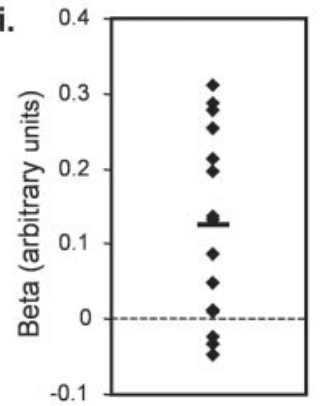

Table 3. Regions activated by aversive olfactory learning

MNI coordinates $(\mathrm{mm})$

\begin{tabular}{|c|c|c|c|c|c|}
\hline \multirow{2}{*}{ Brain region } & & \multirow[b]{2}{*}{ Peak $Z$} & \multirow[b]{2}{*}{$p$ value } \\
\hline & $x$ & $y$ & $z$ & & \\
\hline \multicolumn{6}{|l|}{$\left(\operatorname{avCS}_{U}-\right.$ avCS-$)$} \\
\hline Left lateral OFC & -32 & 52 & -12 & 4.19 & $<0.05^{*}$ \\
\hline Right medial OFC & 14 & 46 & -18 & 3.88 & $<0.001$ \\
\hline \multicolumn{6}{|c|}{$\left(\operatorname{avCS}_{\mathrm{U}}-\right.$ avCS-$) \times$ time: time-dependent decreases } \\
\hline Left nucleus accumbens & -10 & 16 & -12 & 3.10 & $<0.05^{*}$ \\
\hline \multicolumn{6}{|c|}{$\left(\operatorname{avCS}_{U}-\right.$ avCS-$) \times$ time: time-dependent increases } \\
\hline Left medial OFC & -18 & 22 & -14 & 3.99 & $0.058^{*}$ \\
\hline Left temporal pole/piriform cortex & -26 & 6 & -38 & 3.26 & $<0.001$ \\
\hline \multicolumn{6}{|c|}{$\left(\operatorname{avCS}+_{U}-\right.$ appCS-$) \times$ time: time-dependent increases } \\
\hline Left temporal pole/piriform cortex & -30 & 6 & -38 & 4.74 & $<0.001$ \\
\hline
\end{tabular}

* Corrected for small volume of interest.

In our companion paper (Gottfried et al., 2002) on olfactory sensory processing, we showed that odor-evoked activity in OFC was restricted specifically to caudal areas, corresponding anatomically to secondary olfactory cortex in animals (Yarita et al., 1980; Carmichael et al., 1994). This closely overlapped with posterior OFC activations evoked by olfactory learning (Fig. $2 B$ ). In contrast, significant effects within rostral OFC were associated exclusively with olfactory learning (Fig. $2 A$ ) but not with sensory odor processing. On the basis of these observations, we argue that the learning-evoked responses in caudal OFC may reflect modulation of secondary olfactory cortex by higher-order centers. Such influences might help increase the gain in structures that are primarily involved in unimodal olfactory discrimination, thereby promoting more efficient processing of behaviorally salient odors. Conversely, higher-order operations pertaining to learning would be specifically executed in rostral OFC. This idea is bolstered by animal findings suggesting an anatomical hierarchy of orbitofrontal specialization, whereby caudal regions (such as olfactory OFC) converge on medial and anterior territories to permit more complex information processing (Carmichael and Price, 1996; Ongur and Price, 2000). In this regard, neural activity in anterior
OFC has been observed in recent neuroimaging studies of olfactory working memory [at 32, 44, -18 (Dade et al., 2001)] and short- and long-term odor recognition [at 27, 44, -14, and 20, 48, -18 (Dade et al., 2002)], lending further credence to the notion that this region is an important functional component of human olfactory learning.

Recent neuroimaging studies indicate that the neural substrates of reward and punishment may be functionally compartmentalized within medial and lateral OFC, respectively (O'Doherty et al., 2001, 2003; Small et al., 2001). Moreover, our companion study (Gottfried et al., 2002) showed that these dissociations were preserved in response to pleasant and unpleasant odors. In the present context, evidence for medial-lateral distinctions is less compelling but exhibits a comparable trend. Thus, in the main effect of appetitive olfactory learning, multiple activation foci were elicited in medial OFC (Fig. $3 A$ ), whereas responses in lateral OFC were not observed. By comparison, the main effect of aversive learning was associated with neural responses in lateral OFC (Fig. 5A). Although rostromedial OFC was also identified in this contrast, the fact that this area emerged both in the main effect of appetitive learning and in the conjunction analysis sug- 

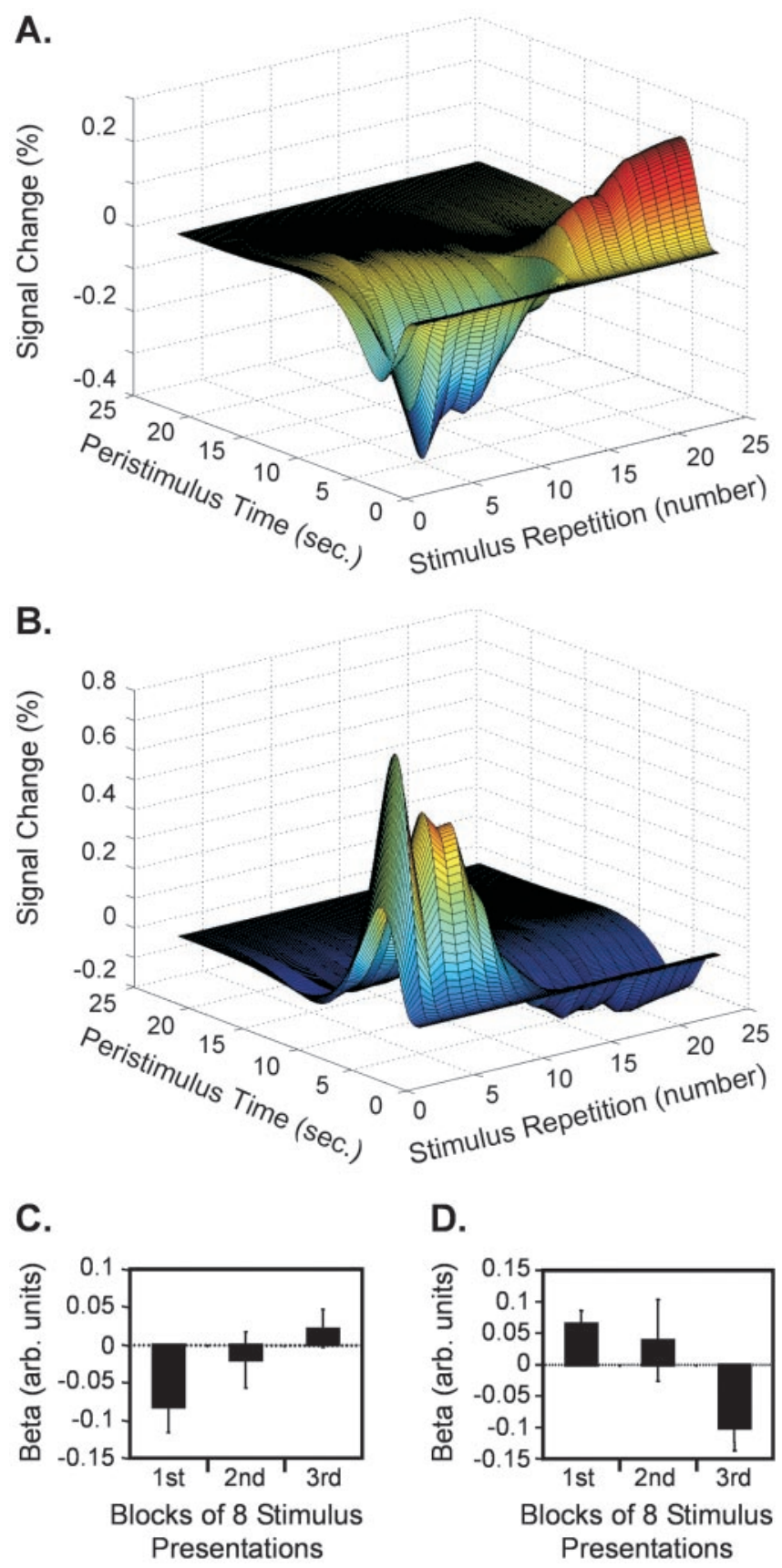

Figure 6. Divergent temporal profiles in nucleus accumbens. $A, C$, Neural activity in nucleus accumbens increased over time during appetitive olfactory learning. $A$, The percentage signal change of the fitted hemodynamic response in nucleus accumbens elicited by an appCS ${ }{ }_{U}$ stimulus (minus the $\mathrm{CS}-$ baseline) is plotted against repetition number for one subject and shows that repeated presentations of appCS ${ }{ }_{\mathrm{U}}$ evoke progressive increases in the response signal. $C$, The contrasts of parameter estimates (appCS $+_{U}-$ appCS - ) (at 14, 16, -12 ) pooled across all subjects (means \pm SEM) are depicted across successive thirds of the experiment and confirm an increasing temporal response profile at the group level. $B, D$, Activity in nucleus accumbens habituated over time during aversive olfactory learning. $B$, The plot of the percentage signal change elicited by avCS ${ }_{U}($ minus $\mathrm{CS}-$ ) reveals a pattern in nucleus accumbens that habituates over repeated stimulus presentations (same subject as in $A$ ). $D$, Similarly, the group response profile of the contrast of parameter estimates (at $-10,16,-12$ ) shows a steady decline over time. gests that it is probably more responsive to nonpunishing aspects of olfactory conditioning.

We found neural responses in nucleus accumbens in the direct

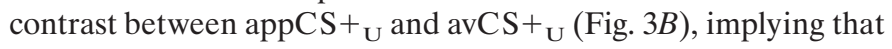
these activations were specifically related to the learning of an olfactory reward. In addition, this differential activity progressively increased with successive appCS ${ }{ }_{U}$ presentations (Figs. 4, 6). Although human neuroimaging studies confirm that nucleus accumbens is modulated by reward-based stimuli (Delgado et al., 2000; O'Doherty et al., 2002; Pagnoni et al., 2002), we believe that our findings are the first to demonstrate that odors drive conditioned reward responses within human mesolimbic structures.

Although nucleus accumbens is a major target of midbrain dopaminergic afferents (Kalivas and Nakamura, 1999), the characterization of dopamine-related effects on evoked BOLD activity is unclear. Nevertheless, a survey of the literature suggests parallels between our data and animal studies of odor learning. For example, single-unit accumbens activity increased in male rats conditioned to associate novel smells with sexually receptive females (West et al., 1992). Similarly, rats administered intraaccumbens cocaine concurrently in the presence of neutral odors developed conditioned place preference for these stimuli (Barr and Wang, 1992).

In primate electrophysiological studies, the output signals of dopamine neurons in the ventral tegmentum are proposed to transmit reward-prediction errors to facilitate appetitive learning (Schultz, 1998). Recent human neuroimaging studies of tastereward anticipation are broadly consistent with this proposal (O'Doherty et al., 2002; Pagnoni et al., 2002). Interestingly, the learning curves generated by temporal-difference models of the dopamine signal (Schultz, 1998) are reminiscent of the temporal profiles observed here in response to olfactory appetitive learning, although our study was not specifically devised to test such hypotheses. On the other hand, conflicting data from lesion, pharmacological, and in vivo microdialysis studies indicate that the animal literature is far from resolved, and whether our findings are ultimately compatible with reward-prediction error or other models (Berridge and Robinson, 1998; Horvitz, 2000) warrants further investigation.

Although neural activity in nucleus accumbens progressively increased in response to appCS+${ }_{U}$, an opposite (decreasing) pattern was seen in response to avCS ${ }_{U}$ (Figs. $5 C, 6$ ). Thus, it appears that the valence of olfactory learning evokes divergent temporal profiles in the same structure. That nucleus accumbens is differentially responsive to positive and negative modes of learning is supported by accumulating evidence highlighting a role for mesolimbic dopamine in aversive processing (Horvitz, 2000). Relevant to our results is the demonstration that dopamine release in ventral striatum decreases as a result of conditioned odor aversion (Abercrombie et al., 1989; Doherty and Gratton, 1992; Besson and Louilot, 1995). In addition, microdialysis studies performed in rodents in vivo indicate that accumbens dopamine increases or decreases in response to rewarding or aversive stimuli, respectively (Di Chiara et al., 1999). However, further work is needed to establish how synaptic dopamine levels relate to fMRI signal change.

Discrete subregions of posterior amygdala were associated exclusively with appetitive learning (Fig. 3C). Responses in dorsomedial areas were sustained over the course of the experiment, whereas ventromedial regions exhibited both sustained and habituating response patterns. The demonstration of different activation loci within the amygdala may reflect functional segrega- 
tion, particularly with respect to habituating and nonhabituating profiles, as shown previously (Morris et al., 2001). Although current fMRI techniques lack sufficient spatial resolution to discern individual amygdala subnuclei, the dorsomedial activation may correspond to the central nucleus of the amygdala (Mai et al., 1997), an area linked to appetitive Pavlovian conditioning in animal lesion studies (Gallagher et al., 1990; Parkinson et al., 2000). In addition, this same region closely overlaps activations evoked by anticipation of pleasant taste (O’Doherty et al., 2002). This anatomical convergence, based on different types of natural rewards, implicates a broad role for posterior dorsal amygdala in appetitive processing. More generally, our data extend recent findings from human studies demonstrating the importance of amygdala to various reward-related learning processes (Hamann et al., 1999; Johnsrude et al., 2000).

We found no significant amygdala activity during aversive olfactory learning or in anticipation of aversive tastes (O'Doherty et al., 2002). These findings are intriguing given its reliable involvement in animal (LeDoux, 2000) and human (Buchel et al., 1998; LaBar et al., 1998) studies of fear conditioning. Indeed, one might have predicted the reverse pattern of results, in which the amygdala was a selective participant in aversive, rather than appetitive, learning. As Cahill and McGaugh (1990) have elegantly shown with aversive taste reinforcers, it is possible that the use of unpleasant odors simply might not be arousing enough to engage amygdala-dependent conditioning (although one might expect this to apply to the pleasant odor as well). Alternately, qualitative differences in the aversive UCS may elicit variable activation patterns. Loud sounds or electric shocks are more likely to produce affective responses approximating fear or startle, shown in diverse species to be potent activators of amygdala (LeDoux, 2000), whereas unpleasant odors may provoke reactions closer to disgust. To this end, faces expressing disgust (as opposed to fear) seem to activate amygdala relatively poorly (Phillips et al., 1997) and may help explain why aversive olfactory learning did not involve this structure.

A remaining possibility is that functional disparities may reflect a unique organization of olfaction, whereby aversive learning would be implemented by other brain regions. Potential candidates include areas of temporal piriform and periamygdaloid cortices, which showed increasing neural activations over time in the main effect of avCS ${ }_{U}$ (relative to avCS-) and in the direct comparison with appCS+ $+_{U}$ (Fig. $5 B$ ). The idea that primary olfactory cortex helps establish salient cross-modal associations is complementary to animal studies indicating that piriform cortex supports learning-related processes (Schoenbaum and Eichenbaum, 1995; Saar et al., 1999; Datiche et al., 2001). Indeed, the detection of learning-evoked activations in both human primary (piriform) and secondary (caudal OFC) olfactory projection sites suggests that these areas should not be considered simply as unimodal sensory relays but as active participants in more complex aspects of odor information processing.

\section{REFERENCES}

Abercrombie ED, Keefe KA, DiFrischia DS, Zigmond MJ (1989) Differential effect of stress on in vivo dopamine release in striatum, nucleus accumbens, and medial frontal cortex. J Neurochem 52: 1655-1658.

Baron RA (1988) Perfume as a tactic of impression management in social and organizational settings. In: Perfumery: the psychology and biology of fragrance (Van Toller S, Dodd GG, eds), pp 91-104. London: Chapman and Hall.

Barr GA, Wang S (1992) Peripheral and central administration of cocaine produce conditioned odor preferences in the infant rat. Brain Res 599:181-185.
Beauchamp GK, Maller O (1977) The development of flavor preferences in humans: a review. In: The chemical senses and nutrition (Kare MR, Maller O, eds), pp 291-311. New York: Academic.

Berridge KC, Robinson TE (1998) What is the role of dopamine in reward: hedonic impact, reward learning, or incentive salience? Brain Res Brain Res Rev 28:309-369.

Besson C, Louilot A (1995) Asymmetrical involvement of mesolimbic dopaminergic neurons in affective perception. Neuroscience 68: 963-968.

Buchel C, Morris J, Dolan RJ, Friston KJ (1998) Brain systems mediating aversive conditioning: an event-related fMRI study. Neuron 20:947-957.

Cahill L, McGaugh JL (1990) Amygdaloid complex lesions differentially affect retention of tasks using appetitive and aversive reinforcement. Behav Neurosci 104:532-543.

Carde RT, Mafra-Neto A (1997) Mechanisms of flight of male moths to pheromone. In: Insect pheromone research: new directions (Carde RT, Minks AK, eds), pp 275-290. New York: Chapman and Hall.

Carmichael ST, Price JL (1996) Connectional networks within the orbital and medial prefrontal cortex of macaque monkeys. J Comp Neurol 371:179-207

Carmichael ST, Clugnet MC, Price JL (1994) Central olfactory connections in the macaque monkey. J Comp Neurol 346:403-434

Cole AD, Bond NW (1983) Olfactory aversion conditioning and overeating: a review and some data. Percept Mot Skills 57:667-678.

Critchley HD, Rolls ET (1996) Olfactory neuronal responses in the primate orbitofrontal cortex: analysis in an olfactory discrimination task. J Neurophysiol 75:1659-1672.

Critchley HD, Mathias CJ, Dolan RJ (2002) Fear conditioning in humans: the influence of awareness and autonomic arousal on functional neuroanatomy. Neuron 33:653-663.

Dade LA, Zatorre RJ, Evans AC, Jones-Gotman M (2001) Working memory in another dimension: functional imaging of human olfactory working memory. NeuroImage 14:650-660.

Dade LA, Zatorre RJ, Jones-Gotman M (2002) Olfactory learning: convergent findings from lesion and brain imaging studies in humans. Brain 125:86-101.

Datiche F, Roullet F, Cattarelli M (2001) Expression of Fos in the piriform cortex after acquisition of olfactory learning: an immunohistochemical study in the rat. Brain Res Bull 55:95-99.

Deichmann R, Turner R (2002) Improvement of local BOLD sensitivities in the presence of susceptibility gradients by using tilted slices. Proc Int Soc Magn Reson Med 10:1414.

Delgado MR, Nystrom LE, Fissell C, Noll DC, Fiez JA (2000) Tracking the hemodynamic responses to reward and punishment in the striatum. J Neurophysiol 84:3072-3077.

Di Chiara G, Loddo P, Tanda G (1999) Reciprocal changes in prefrontal and limbic dopamine responsiveness to aversive and rewarding stimuli after chronic mild stress: implications for the psychobiology of depression. Biol Psychiatry 46:1624-1633.

Doherty MD, Gratton A (1992) High-speed chronoamperometric measurements of mesolimbic and nigrostriatal dopamine release associated with repeated daily stress. Brain Res 586:295-302.

Friston KJ, Ashburner J, Frith CD, Poline J-B, Heather JD, Frackowiak RSJ (1995a) Spatial registration and normalization of images. Hum Brain Mapp 2:165-189.

Friston KJ, Holmes AP, Worsley KJ, Poline J-P, Frith CD, Frackowiak RSJ (1995b) Statistical parametric maps in functional imaging: a general linear approach. Hum Brain Mapp 2:189-210.

Frohwirth RA, Foreyt JP (1978) Aversive conditioning treatment of overweight. Behav Res 9:861-872.

Gallagher M, Graham PW, Holland PC (1990) The amygdala central nucleus and appetitive Pavlovian conditioning: lesions impair one class of conditioned behavior. J Neurosci 10:1906-1911.

Gottfried JA, Deichmann R, Winston JS, Dolan RJ (2002) Functional heterogeneity in human olfactory cortex: an event-related fMRI study. J Neurosci 22:10819-10828.

Hamann SB, Ely TD, Grafton ST, Kilts CD (1999) Amygdala activity related to enhanced memory for pleasant and aversive stimuli. Nat Neurosci 2:289-293.

Hermann C, Ziegler S, Birbaumer N, Flor H (2000) Pavlovian aversive and appetitive odor conditioning in humans: subjective, peripheral, and electrocortical changes. Exp Brain Res 132:203-215.

Horvitz JC (2000) Mesolimbocortical and nigrostriatal dopamine responses to salient non-reward events. Neuroscience 96:651-656.

Johnsrude IS, Owen AM, White NM, Xhao WV, Bohbot V (2000) Impaired preference conditioning after anterior temporal lobe resection in humans. J Neurosci 20:2649-2656.

Kalivas PW, Nakamura M (1999) Neural systems for behavioral activation and reward. Curr Opin Neurobiol 9:223-227.

LaBar KS, Gatenby JC, Gore JC, LeDoux JE, Phelps EA (1998) Human amygdala activation during conditioned fear acquisition and extinction: a mixed-trial fMRI study. Neuron 20:937-945.

LeDoux JE (2000) Emotion circuits in the brain. Annu Rev Neurosci 23:155-184. 
Lorig TS, Elmes DG, Zald DH, Pardo JV (1999) A computer-controlled olfactometer for fMRI and electrophysiological studies of olfaction. Behav Res Methods Instrum Comput 31:370-375.

MacFarlane A (1975) Olfaction in the development of social preferences in the human neonate. Ciba Found Symp 33:103-117.

Mai JK, Assheuer J, Paxinos G (1997) Atlas of the human brain. San Diego: Academic.

Morris JS, Buchel C, Dolan RJ (2001) Parallel neural responses in amygdala subregions and sensory cortex during implicit fear conditioning. NeuroImage 13:1044-1052.

O'Doherty J, Rolls ET, Francis S, Bowtell R, McGlone F, Kobal G, Renner B, Ahne G (2000) Sensory-specific satiety-related olfactory activation of the human orbitofrontal cortex. NeuroReport 11:893-897.

O'Doherty J, Kringelbach ML, Rolls ET, Hornak J, Andrews C (2001) Abstract reward and punishment representations in the human orbitofrontal cortex. Nat Neurosci 4:95-102.

O'Doherty J, Deichmann R, Critchley HD, Dolan RJ (2002) Neural responses during anticipation of a primary taste reward. Neuron 33:815-826.

O'Doherty J, Winston JS, Critchley H, Perrett D, Burt DM, Dolan RJ (2003) Beauty in a smile: the role of medial orbitofrontal cortex in facial attractiveness. Neuropsychologia 41:147-155.

Ongur D, Price JL (2000) The organization of networks within the orbital and medial prefrontal cortex of rats, monkeys and humans. Cereb Cortex 10:206-219.

Pagnoni G, Zink CF, Montague PR, Berns GS (2002) Activity in human ventral striatum locked to errors of reward prediction. Nat Neurosci 5:97-98.

Parkinson JA, Robbins TW, Everitt BJ (2000) Dissociable roles of the central and basolateral amygdala in appetitive emotional learning. Eur J Neurosci 12:405-413.

Phillips ML, Young AW, Senior C, Brammer M, Andrew C, Calder AJ, Bullmore ET, Perrett DI, Rowland D, Williams SC, Gray JA, David AS (1997) A specific neural substrate for perceiving facial expressions of disgust. Nature 389:495-498.

Potter H, Butters N (1980) An assessment of olfactory deficits in patients with damage to prefrontal cortex. Neuropsychologia 18:621-628.

Price CJ, Friston KJ (1997) Cognitive conjunction: a new approach to brain activation experiments. NeuroImage 5:261-270.

Royet JP, Hudry J, Zald DH, Godinot D, Gregoire MC, Lavenne F,
Costes N, Holley A (2001) Functional neuroanatomy of different olfactory judgments. NeuroImage 13:506-519.

Saar D, Grossman Y, Barkai E (1999) Reduced synaptic facilitation between pyramidal neurons in the piriform cortex after odor learning. J Neurosci 19:8616-8622.

Savic I, Gulyas B, Larsson M, Roland P (2000) Olfactory functions are mediated by parallel and hierarchical processing. Neuron 26:735-745.

Schiffman SS (1974) Physicochemical correlates of olfactory quality. Science 185:112-117.

Schoenbaum G, Eichenbaum H (1995) Information coding in the rodent prefrontal cortex. I. Single-neuron activity in orbitofrontal cortex compared with that in pyriform cortex. J Neurophysiol 74:733-750.

Schoenbaum G, Chiba AA, Gallagher M (1998) Orbitofrontal cortex and basolateral amygdala encode expected outcomes during learning. Nat Neurosci 1:155-159.

Schultz W (1998) Predictive reward signal of dopamine neurons. J Neurophysiol 80:1-27.

Small DM, Zatorre RJ, Dagher A, Evans AC, Jones-Gotman M (2001) Changes in brain activity related to eating chocolate: from pleasure to aversion. Brain 124:1720-1733.

Stern K, McClintock MK (1998) Regulation of ovulation by human pheromones. Nature 392:177-179.

Todrank J, Byrnes D, Wrzesniewski A, Rozin P (1995) Odors can change preferences for people in photographs: a cross-modal evaluative conditioning study with olfactory USs and visual CSs. Learn Motiv 26:116-140.

West CH, Clancy AN, Michael RP (1992) Enhanced responses of nucleus accumbens neurons in male rats to novel odors associated with sexually receptive females. Brain Res 585:49-55.

Worsley KJ, Marrett S, Neelin P, Vandal AC, Friston KJ, Evans AC (1996) A unified statistical approach for determining significant voxels in images of cerebral activation. Hum Brain Mapp 4:58-73.

Yarita H, Ino M, Tanabe T, Kogure S, Takagi SF (1980) A transthalamic olfactory pathway to orbitofrontal cortex in the monkey. J Neurophysiol 43:69-85.

Zald DH, Pardo JV (2000) Functional neuroimaging of the olfactory system in humans. Int J Psychophysiol 36:165-181.

Zatorre RJ, Jones-Gotman M (1991) Human olfactory discrimination after unilateral frontal or temporal lobectomy. Brain 114:71-84. 